\title{
Tumor formation and drug resistance properties of human glioblastoma side population cells
}

\author{
DONG CHEN \\ Department of Neurosurgery, Tianjin Huanhu Hospital, Tianjin 300060, P.R. China
}

Received March 2, 2014; Accepted November 25, 2014

DOI: $10.3892 / \mathrm{mmr} .2015 .3279$

\begin{abstract}
Accumulating evidence has demonstrated that the presence of a subset of cells in several types of brain tumor, termed brain cancer stem cells, are responsible for tumor recurrence following chemotherapy or radiotherapy. The isolation and characterization of side population (SP) cells in several types of solid tumor using Hoechst dye has become a powerful technique for obtaining cancer stem cells (CSCs). In the present study, cancer stem-like SP cells were isolated from the human glioblastoma cell line MG-12 using the Hoechst 33342 dye exclusion technique. Flow cytometric analysis revealed that the cell line MG-12 contained 3.2\% SP cells, which was reduced to $0.5 \%$ in the presence of verapamil, an inhibitor of ATPase-binding cassette (ABC) transporters. Reverse-transcription quantitative polymerase chain reaction analysis revealed that the isolated SP cells exhibited increased expression of stem cell markers, including Nestin, Notch 1, octamer-binding transcription factor $4(O c t-4)$, epithelial cell adhesion molecule (EpCAM) and also $\mathrm{ABC}$ transporter protein $A B C G 2$. Additionally, using western blot analysis it was demonstrated that SP cells exhibit positivity and have a higher expression of CD133,CD44,EpCAM, Oct-4 and B-cell lymphoma 2. Furthermore, it was demonstrated that the isolated SP cells undergo rapid proliferation, have a high propensity to form tumor spheres and also have a high survival rate following treatment with 5-fluorouracil. Therefore, the present findings suggest that SP cells of the glioblastoma MG-12 cell line share characteristics of CSCs. Therefore, the increased expression of stem cell markers and $A B C G 2$ protein may interact with each other and be responsible for drug and apoptotic resistance, tumor recurrence and invasion.
\end{abstract}

Correspondence to: Dr Dong Chen, Department of Neurosurgery, Tianjin Huanhu Hospital, 122 Qixiangtai Road, Hexi, Tianjin 300060, P.R. China

E-mail: chendong060@gmail.com

Key words: cancer stem cells, glioblastoma, side population, multidrug resistance, chemotherapy

\section{Introduction}

The most frequently occurring malignant brain tumor is glioblastoma, which has a median survival time of less than one year (World Health Organization grade IV). Despite advances in treatment strategy, the prognosis of glioblastoma patients has not substantially improved and tumor recurrence frequently occurs in patients following treatment (1-3). Previous studies in several types of solid tumor, including brain tumors, reported that the presence of cancer stem cells (CSCs) is responsible for treatment failure and tumor recurrence (4-8). Brain cancer stem cells (BCSCs) are capable of self-renewal and express neural stem cell surface markers, including CD133 and Nestin. In addition, CSCs are able to form neurospheres, differentiate into other cell types, including neurons, astrocytes and oligodendrocytes, have the potential to initiate tumor growth and also exhibit multidrug and apoptotic resistance $(9,10)$. The conventional treatment strategies target only the bulk of the tumor cells, leaving the CSCs unaffected, which results in treatment failure and is responsible for minimal residual disease $(11,12)$. Therefore, the development of new therapeutic strategies that effectively target BCSCs is crucial. Several methods have been proposed for the isolation of BCSCs from brain tumors or glioma. These include the isolation of glioma tissues as spheres in serum-free medium, sorting of BCSCs with antibodies for stem cell surface markers and Hoechst 33342 dye exclusion by flow cytometry $(3,13,14)$. It has previously been reported in several types of solid tumor that the Hoechst 33342 dye exclusion is a powerful and valuable technique for isolating CSCs $(4,15,16)$.

Cells that exclude Hoechst 33342 dye are termed side population (SP) cells. These cells share characteristics with BCSCs, including the ability to initiate tumor growth following treatment, such as chemotherapy or radiotherapy and the expression of stem cells genes, including CD133, $C D 44, C D 34, C D 29$ and $C D 24$. Furthermore, SP cells also have a higher expression of ATPase binding cassette (ABC) transporters, including $A B C B 1$ [multidrug resistance transporter $1(M D R 1)$ ], $A B C C 1$ and $A B C G 2$ [breakpoint cluster region pseudogene 1 (BCRP1)], which contribute to multidrug resistance. Therefore, determination of key SP cells in the tumor population that are able to maintain the tumor may provide new insight into the mechanism of brain tumorigenesis and assist in tracing the tumor cell of origin 
for providing effective treatment. Thus, in the present study, SP cells were isolated and characterized from the human glioblastoma cell line MG-12.

\section{Materials and methods}

Cell line and cell culture. The human glioblastoma MG-12 cell line was established from a patient with malignant glioblastoma (Grade IV). The tumor samples were collected from the patient in accordance with the ethical principles approved by the Department of Neurosurgery, Tianjin Huanhu Hospital, Tianjin, China and written informed consent was obtained. Cell lines were maintained in Dulbecco's modified Eagle's medium (DMEM), which was supplemented with $10 \%$ fetal bovine serum, $100 \mathrm{U} / \mathrm{ml}$ penicillin $\mathrm{G}$ and $100 \mathrm{lg} / \mathrm{ml}$ streptomycin. To generate glioma spheres, cells were cultured in a neurosphere culture medium (NSP medium) consisting of neurobasal medium supplemented with human recombinant endothelial growth factor $(20 \mathrm{ng} / \mathrm{ml})$ human recombinant fibroblast growth factor $2(20 \mathrm{ng} / \mathrm{ml}), \mathrm{B} 27$, heparin $(10 \mathrm{ng} / \mathrm{ml})$ and human recombinant leukemia inhibitory factor (10 ng/ $\mathrm{ml})$. All reagents and chemicals were purchased from Sigma-Aldrich, Shanghai, China.

Study group. Group I, control-MG-12 cells+Hoechst 33342 dye $(n=6)$; Group II, drug treated-MG-12 cells+verapamil+Hoechst 33342 dye $(n=6)$.

Labeling with Hoechst 33342. Using a hemocytometer, $\sim 10^{6}$ cells $/ \mathrm{ml}$ in $10 \%$ DMEM were labeled with Hoechst 33342 (Sigma, St. Louis, MO, USA) stock bis-benzimide $(5 \mu \mathrm{l} / \mathrm{ml})$ either with dye alone or in combination with drug treatment (verapamil, $0.8 \mu \mathrm{l} / \mathrm{ml}$ ). After 90 min incubation in a water bath at $37^{\circ} \mathrm{C}$, cells were subjected to centrifugation at 2,500 $\mathrm{x} \mathrm{g}$ for $10 \mathrm{~min}$ at $4^{\circ} \mathrm{C}$ and resuspended in $500 \mu 1$ of Hank's balanced salt solution containing $10 \mathrm{mM}$ 4-(2-hydroxyethyl)-1-piperazineethanesulfonic acid. Finally, cells were counterstained with propidium iodide $(2 \mu \mathrm{g} /$ $\mathrm{ml}$ sample) at $4^{\circ} \mathrm{C}$ to exclude dead cells. Cells were filtered through a $50 \mu \mathrm{m}$ nylon mesh (BD Biosciences, Franklin Lakes, NJ, USA) to remove cell clumps into labeled fluorescence-activated cell sorting (FACS) tubes. The SP cells and main population (non-SP) cells were sorted using a flow cytometer (FACS Aria II; BD Biosciences). The Hoechst 33342 dye was excited at $355 \mathrm{~nm}$ and its dual-wavelength fluorescence was analyzed (blue, $450 \mathrm{~nm}$; red, $675 \mathrm{~nm}$ ).

Sphere formation assay. The sphere formation assays for the sorted SP and non-SP cells were performed as described previously (3). SP and non-SP cells sorted from the MG-12 cell line were seeded at a low density of 20 cells/ 1 and the number of generated spheres was counted after 8 days of culture.

In vitro proliferation activity assay. The sorted SP and non-SP cells were seeded in a 96-well plate at $2 \times 10^{6}$ cells/well $(n=4)$ and incubated in a $\mathrm{CO}_{2}$ incubator. Cell proliferation activity was measured each day for 6 days. Each well was supplemented with cell counting kit-8 (CCK-8) solution $(10 \mu \mathrm{l})$ and incubated in a $\mathrm{CO}_{2}$ incubator for 2-3 $\mathrm{h}$. The optical density
(OD) was determined at $450 \mathrm{~nm}$. These data were used to calculate cell growth graphs based on the mean value of $\mathrm{OD}_{450}$ and standard deviation values for each well.

Cell resistance assay. The sorted SP and non-SP cells were seeded in 96-well plates at a concentration of $1 \times 10^{3}$ cells/plate. After $24 \mathrm{~h}, 10 \mu \mathrm{g} / \mathrm{ml} 5$-fluorouracil (5-FU) was added to all cultures and incubated for $48 \mathrm{~h}$. Subsequently, each well was supplemented with CCK-8 solution $(10 \mu \mathrm{l})$ and the plates were incubated for $3 \mathrm{~h}$. The mean value of $\mathrm{OD}_{450}$ obtained was presented as a graph as described previously (15). Cell resistance in the two groups was calculated using the following formula: Cell resistance rate $(\%)=($ experimental group $\mathrm{OD}_{450}$ value/control group $\mathrm{OD}_{450}$ value) x 100 .

Reverse-transcription quantitative polymerase chain reaction (RT-qPCR). Total RNA was isolated from FACS-sorted SP and non-SP cells using the Ambion RNAqueous ${ }^{\circledR}$-Micro kit (Applied Biosystems, Warrington, UK). cDNA was synthesized using the Bioline cDNA synthesis kit (Bioline, London, UK). RT-qPCR was performed using 2-3 $\mu 1$ cDNA and 2X TaqMan Gene Expression Mastermix (Applied Biosystems) in $50 \mu 1$ reaction volumes. Glyceraldehyde-3-phosphate dehydrogenase $(G A P D H)$ was used as the standard endogenous expression. The primers were designed to encompass an exon junction to prevent templating from possibly contaminated genomic DNA. The primer sequences used were as follows: $A B C G 2$, forward 5'-AGC TGC AAG GAA AGA TCC AA-3' and reverse 5'-TCC AGA CAC ACC ACG GAT AA; octamer-binding transcription factor $4(O c t-4)$, forward 5'-ATC CTG GGG GTT CTA TTT GG-3' and reverse 5'-CTC CAG GTT GCC TCT CAC TC-3'); epithelial cell adhesion molecule (EpCAM), forward 5'-CTG CCA AAT GTT TGG TGA TG and reverse 5'-ACG CGT TGT GAT CTC CTT CT-3'; Nestin, forward 5'-TGG CTC AGA GGA AGA GTC TGA-3' and reverse 5'-TCC CCC ATT TAC ATG CTG TGA-3'; Notch 1, forward 5'-CAG GCA ATC CGA GGA CTA TG-3' and reverse 5'-CAG GCG TGT TGT TCT CAC AG-3' and GAPDH, forward 5'-ATG TCG TGG AGT CTA CTG GC-3' and reverse 5'-TGA CCT TGC CCA CAG CCT TG-3'. The amplified products were separated by electrophoresis on ethidium bromide-stained 1.2\% agarose gels. Band intensity was measured using Image J software, version 1.4 (National Institutes of Health, Bethesda, MA, USA) from three independent experiments.

Biochemistry. For western blot analysis, proteins were extracted from the SP and non-SP cells and protein concentration was determined using the Bradford assay. Blots were probed with the monoclonal primary antibodies, 1:1,000 [rabbit anti-human $A B C G 2, C D 133, C D 44$, B-cell lymphoma $2(B c l-2)$ and Actin], the monoclonal secondary antibody $(1: 10,000$; goat anti-rabbit IgG with alkaline phosphatase markers) purchased from Santa Cruz Biotechnology, Inc., (Dallas, TX, USA). and a chemiluminescence reagent.

Statistical analysis. A one-way analysis of variance was performed to determine the significance between different treatment groups and individual Student's t-tests were performed to compare the effect of different treatments 
between the SP and non-SP populations. $\mathrm{P}<0.05$ was considered to indicate a statistically significant difference.

\section{Results}

Isolation of SP cells from the human glioma cell line MG-12. The malignant human glioblastoma cell line was analyzed for the presence of SP cells using Hoechst 33342 dye, which is a DNA binding dye. Using FACS, the live cell population was selected against propidium iodide, which was used to exclude dead cells from the samples. The presence of a distinct SP cell population (P2) was identified towards the SP-violet region of the dot plot of the FACS profile (Fig. 1A), which accounts for $3.2 \%$ of the total cell population. The exclusion of Hoechst 33342 by SP cells is an active process, which involves MDR 1 , a member of the ABC transporter family. Since verapamil inhibits the activity of the Hoechst 33342 transporters, the SP cell fraction was decreased in the presence of verapamil (17). Therefore, following treatment with verapamil, the SP cells (P2 gated) were reduced from 3.2 to $0.5 \%$ (Fig. 1B). The present findings suggest that the sorted SP cells are highly resistant to multidrug uptake, which may be due to the overexpression of $\mathrm{ABC}$ transporters and therefore in the presence of verapamil, the percentage of SP cells is significantly reduced.

Sphere formation and drug resistance properties of SP cells. The sorted SP cells and non-SP cells were subsequently subjected to sphere formation assays. In order to compare their self-renewing capacities, SP cells and non-SP cells were cultured in NSP medium at a low density $(20$ cells $/ \mathrm{ml})$ and analyzed for sphere formation. The SP cells were able to grow and started to form spheres by day 5 (Fig. 2A). The floating spheres in suspension, which were generated from single cells of the MG-12 cell line increased in size over time (Fig. 2A; day 9 and 14). However, non-SP cells were unable to propagate under these conditions. The number of spheres generated after 8 days was also counted. The number of spheres generated by SP cells was significantly higher than that produced by non-SP cells (Fig. 2B).

The sorted SP and non-SP cells were further subjected to in vitro cell proliferation and drug resistance assays. The SP cells of the MG-12 cell line of human glioblastoma origin exhibited increased cell proliferation starting from day 3 and became confluent on day 6 when compared with non-SP cells (Fig. 3A). Subsequently, the SP cells were analyzed for drug resistance. Following treatment with $10 \mu \mathrm{g} / \mathrm{ml} 5-\mathrm{FU}$, the survival rate of SP cells (92\%) was significantly higher (Fig. 3B) compared with non-SP cells (49\%). Therefore, the present data suggest that the sorted glioblastoma SP cells are highly resistant to drug treatment and have a high proliferation rate.

Expression of stem cell genes in MG-12 SP cells. Several studies have demonstrated that overexpression of the MDRI transporter, particularly $A B C G 2$, contributes to Hoechst dye expulsion and the drug resistance properties of SP cells in several types of solid tumor $(18,19)$. In addition, the expression of stem cell genes, including Nestin and Notch 1 have been implicated in various cancer cells, including BCSCs $(9,20,21)$. Therefore, the gene expression of $A B C G 2$,
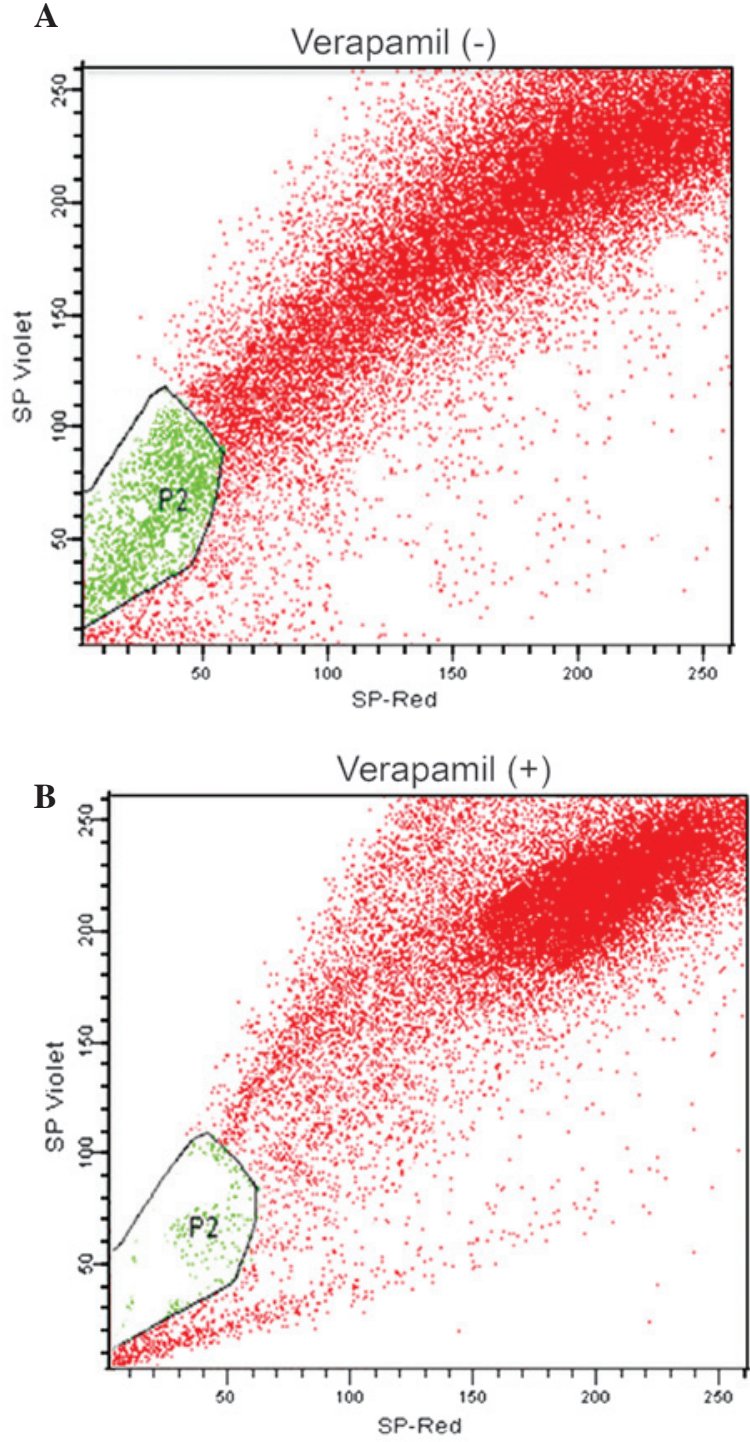

Figure 1. Identification and isolation of SP cells from human glioblastoma MG-12 cells. (A) Cells labeled with Hoechst 33342 showing $3.2 \%$ of SP cells in the P2 gated region. (B) Following treatment with verapamil, the SP cells reduced to $0.5 \%$ ( $\mathrm{P} 2$ gated). $\mathrm{SP}$, side population.

Nestin, Notch 1, EpCAM and Oct-4 was analyzed in SP and non-SP cells from the MG-12 cell line. RT-qPCR analysis revealed that $A B C G 2$, Nestin and EPCAM were more highly expressed in SP cells than in non-SP cells (Fig. 4A). Additionally, the genes Notch 1 and Oct-4 were expressed highly in SP cells whereas the expression levels were almost null in non-SP cells (Fig. 4A). The quantification graph clearly demonstrates that the expression levels of these genes were significantly higher in SP cells compared with non-SP cells (Fig. 4B). The housekeeping gene GAPDH was used as a control. Subsequently, western blot analysis revealed that the protein expression of $A B C G 2, C D 133, C D 44$ and $B c l-2$ was significantly increased in SP cells, whereas these proteins levels were significantly reduced in non-SP cells (Fig. 5). These findings clearly demonstrate that elevated expression of $A B C G 2$ and other stem cell and anti-apoptotic genes/proteins are possibly responsible for the drug/apoptotic resistance, self-renewal capacity and rapid proliferation of cancer cells. 
A
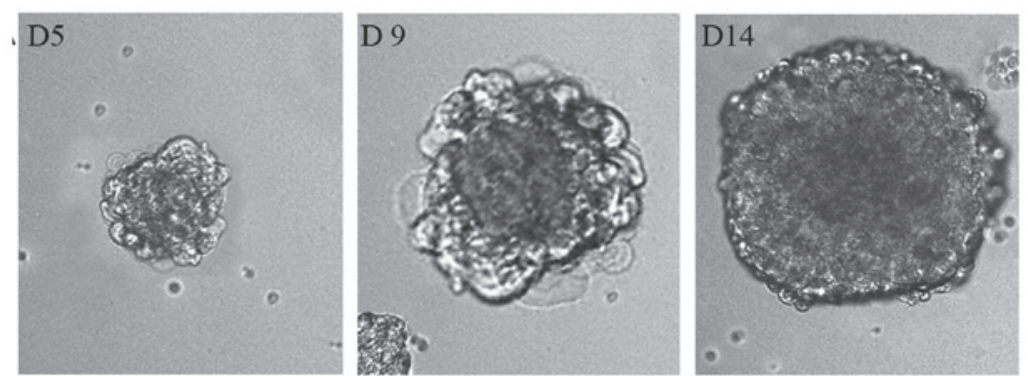

B

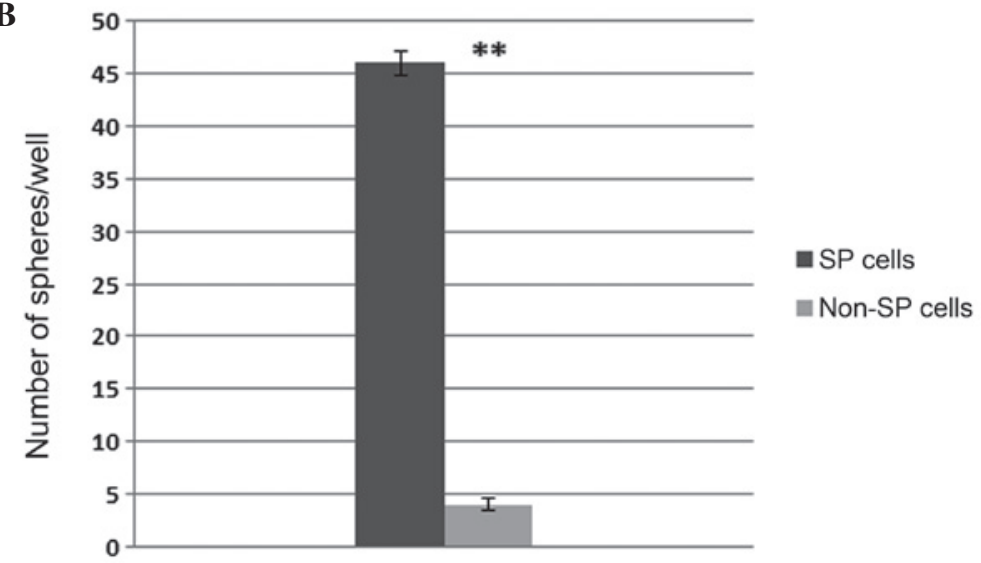

Figure 2. Representative phase contrast photomicrographs (magnification, x200) of tumor spheres from SP cells cultured in NSP medium. (A) SP cells were able to grow, forming spheres at day 5. Sphere formation in suspension generated from single cells increased in size over day 9 and day 14. (B) Quantification of sphere forming capacities of sorted SP and non-SP cells, were seeded into 96-well plates at low cell density (20 cells/l) in neurosphere culture medium containing epidermal growth factor and fibroblast growth factor 2 . After 7 days of culturing, the number of spheres formed by SP cells was significantly greater than that in the non-SP cells. The bar represents standard deviation. ${ }^{* *} \mathrm{P}<0.0001$ vs. non-SP cells. SP, side population.

A

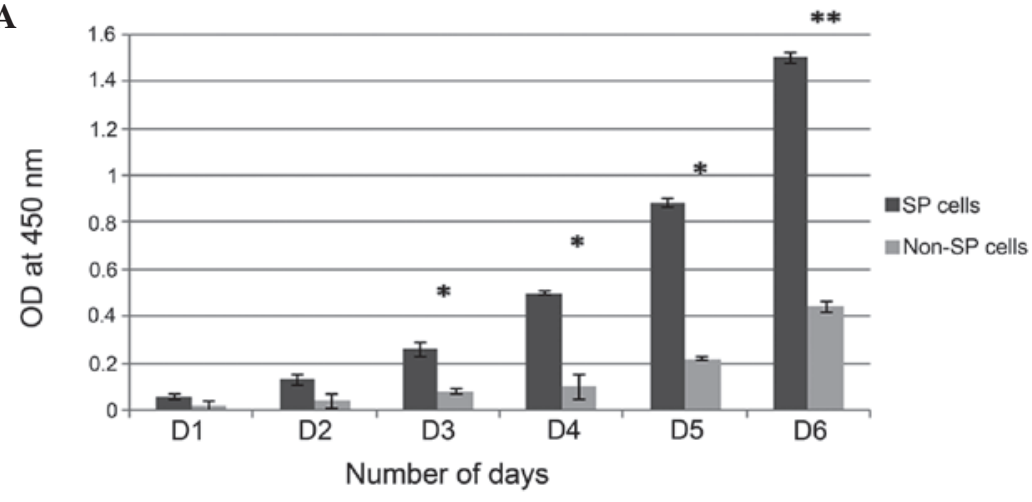

B

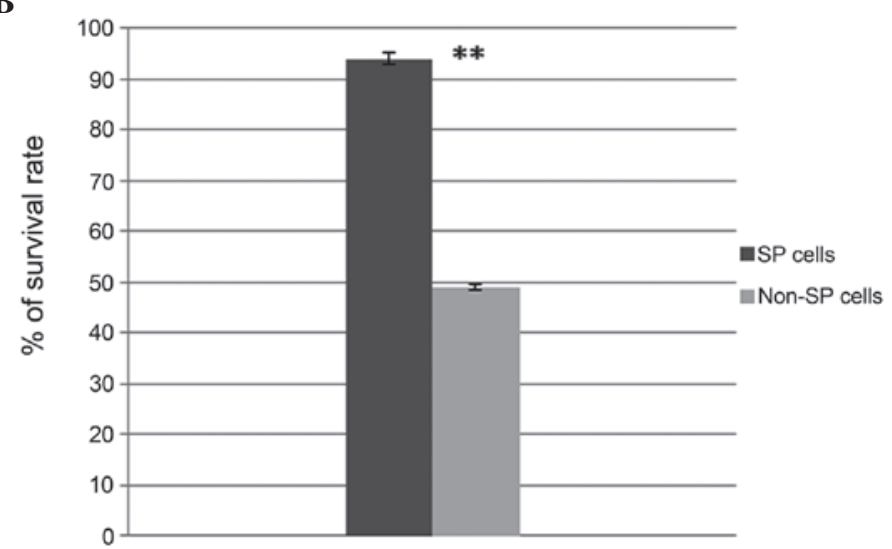

Figure 3. Cell proliferation and drug resistance assays. (A) Cell proliferation rate for SP and non-SP cells. The OD was measured at $450 \mathrm{~nm}$. SP cells underwent rapid proliferation compared with non-SP cells. (B) SP cells exhibited high resistance to 5-fluorouracil whereas the non-SP cells were sensitive to 5-fluorouracil. Bar represents standard deviation. ${ }^{*} \mathrm{P}<0.05$ and ${ }^{* *} \mathrm{P}<0.01$ compared wtih non-SP cells. SP, side population; OD, optical density. 
A

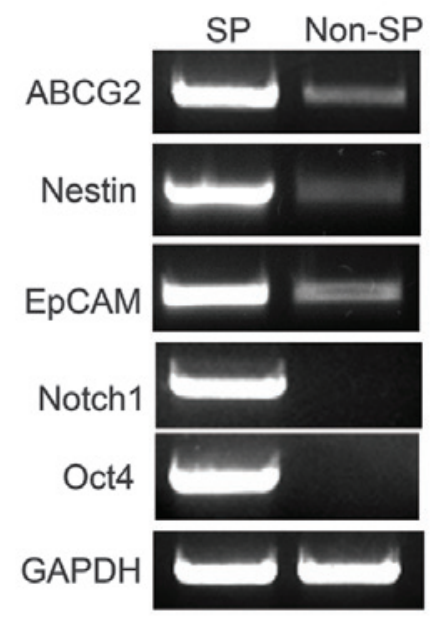

B

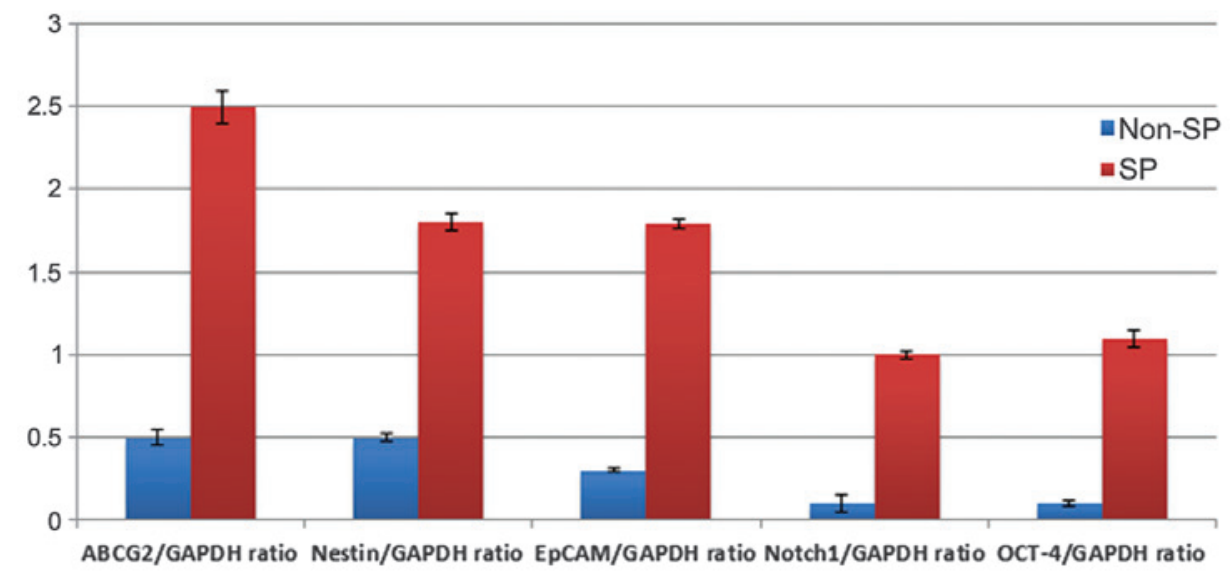

Figure 4. Gene expression analysis of SP cells by RT-qPCR. (A) The gene expression of ABCG2, Notch 1, EpCAM, Oct-4 and Nestin was significantly higher in MG-12 SP cells compared with non-SP cells. GAPDH was used as a control. (B) Quantification graph of the expression level for the above mentioned genes detected by reverse transcription quantitative polymerase chain reaction. Data are presented as the mean \pm standard deviation of three independent experiments, each performed in duplicate. SP, side population; ABCG2, ATP-binding cassette sub-family G member 2; EpCAM, epithelial cell adhesion molecule; OCT-4, octamer-binding transcription factor.

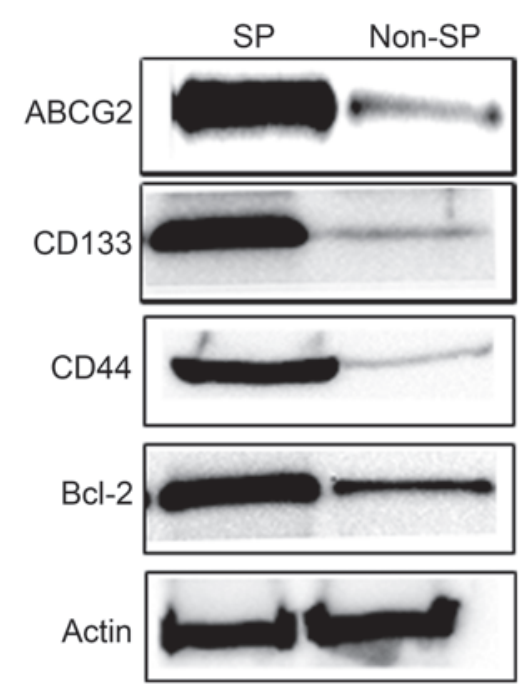

Figure 5. Western blot analysis of protein expression levels in SP and non-SP cells. Equal concentrations of protein were loaded per lane. Actin was used as a loading control. SP, side population; $A B C G 2$, ATP-binding cassette sub-family $\mathrm{G}$ member 2; Bcl-2, B-cell lymphoma 2.

\section{Discussion}

Previously, it has been identified that cancer treatment failure may be due to the persistence of CSCs, which possess multiple characteristics associated with stem cells, including the capacity for self-renewal, high tumorigenicity, a high differentiation potential as well as multidrug and apoptotic resistance $(5,22-24)$. These CSCs evade the treatment regimen and may be responsible for minimal residual disease. Therefore, CSCs are the main target for eradicating cancerous growth completely. Cells that exclude Hoechst 33342 dye are defined as SP cells, which share a number of characteristics with CSCs, specifically tumor initiating capacity, expression of stem cell surface markers and resistance to chemotherapeutic drugs $(22,25)$. In addition, SP cells are enriched in $\mathrm{ABC}$ transporters, including $A B C B 1$ (MDRl), $A B C C 1$ and $A B C G 2$ (BCRP1), which contribute to multidrug resistance (26). These SP cells were identified and characterized in several types of solid tumor and cancer cell lines based on Hoechst 33342 dye efflux by FACS $(5,27)$. In the present study, cancer stem-like SP cells were isolated from human glioblastoma cell lines using the Hoechst 33342 dye exclusion method. FACS analysis revealed that the human glioblastoma MG-12 cell line contained 3.2\% SP cells, whose presence was markedly reduced to $0.5 \%$ when treated with verapamil. Previous studies have demonstrated that SP cells account for $1-2.5 \%$ of cells in the majority of glioblastoma cell lines $(3,5)$. Furthermore, it was demonstrated that sorted SP cells were highly capable of self-renewal as they were able to form spheres in NSP medium and had an increased capacity to proliferate in vitro. The drug resistance assays clearly demonstrated that MG-12 SP cells are highly resistant to 5-FU, therefore they have an increased survival rate, whereas the non-SP cells were sensitive to 5-FU.

Overexpression of $A B C G 2$ may be responsible for multidrug resistance and involves the direct downstream targeting of Notch 1, which promotes the expression of Nestin, a neural stem cell marker, in glioma cells (28). The Notch and Nestin pathway has been observed to promote the survival rate and proliferation of neural stem cells (29). Using RT-qPCR analysis, it was also observed that the gene expression of $A B C G 2$, Nestin, EpCAM, Oct-4 and Notch 1 in SP cells was increased compared with non-SP cells. It was also observed that the isolated MG-12 SP cells exhibited positivity and elevated protein expression for stem cell surface proteins, including CD133, CD44, EpCAM and Oct-4. In line with the current findings, it was observed that $C D 44^{+}$cancer stem cells in head and neck squamous cell carcinomas were highly tumorigenic and able to propagate tumor formation in mice (16). In addition, the expression of $O c t$ - 4 in human gliomas enables self renewal and promotes colony formation in glioma cells (30). Thus, the present results suggest that elevated expression of $C D 133$, 
$C D 44, A B C G 2$ and $B c l-2$ in SP cells may co-operatively act as crucial factors in drug and cell death resistance, proliferation of cancer cells and tumor invasion.

In conclusion, it was identified that MG-12, a human glioblastoma cell line, contained a high percentage of SP cells that possess the properties of BCSCs, including self-renewal, increased cell proliferation rate, the ability to form spheres and high drug resistance. The elevated expression of $A B C G 2$, Nestin, Notch 1, Oct-4 and EpCAM in SP cells may interact with each other and function collectively to contribute to drug resistance, resistance to apoptosis and the enhanced survival rate of SP cells. However, the mechanisms underlying the Notch signaling pathways and the cascade of downstream events in cancer stem cells remain to be elucidated. Therefore, the identification and characterization of SP cells provide a strategy to design novel therapeutic drugs, which target BCSCs in order to prevent tumor recurrence.

\section{References}

1. Holland EC: Gliomagenesis: genetic alterations and mouse models. Nat Rev Genet 2: 120-129, 2001.

2. Kleihues P, Louis DN, Scheithauer BW, et al: The WHO classification of tumors of the nervous system. J Neuropathol Exp Neurol 61: 215-225, 2002.

3. Fukaya R, Ohta S, Yamaguchi M, et al: Isolation of cancer stem-like cells from a side population of a human glioblastoma cell line, SK-MG-1. Cancer Lett 291: 150-157, 2010.

4. Hirschmann-Jax C, Foster AE, Wulf GG, et al: A distinct 'side population of cells' with high drug efflux capacity in human tumor cells. Proc Natl Acad Sci USA 101: 14228-14233, 2004.

5. Patrawala L, Calhoun T, Schneider-Broussard R, Zhou J, Claypool K and Tang DG: Side population is enriched in tumorigenic, stem-like cancer cells, whereas $A B C G 2+$ and $A B C G 2$ - cancer cells are similarly tumorigenic. Cancer Res 65: 6207-6219, 2005.

6. Lee TK, Castilho A, Ma S and Ng IO: Liver cancer stem cells: implications for a new therapeutic target. Liver Int 29: 955-965, 2009.

7. Al-Hajj M, Wicha MS, Benito-Hernandez A, Morrison SJ and Clarke MF: Prospective identification of tumorigenic breast cancer cells. Proc Natl Acad Sci USA 100: 3983-3988, 2003.

8. Dalerba P, Dylla SJ, Park IK, Liu R, Wang X, Cho RW, et al: Phenotypic characterization of human colorectal cancer stem cells. Proc Natl Acad Sci USA 104: 10158-10163, 2007.

9. Singh SK, Clarke ID, Terasaki M, et al: Identification of a cancer stem cell in human brain tumors, Cancer Res 63: 5821-5828, 2003.

10. Singh SK1, Hawkins C, Clarke ID, et al: Identification of human brain tumour initiating cells. Nature 432: 396-401, 2004.

11. Challen GA and Little MH: A side order of stem cells: the SP phenotype. Stem Cells 24: 3-12, 2006.

12. Ramachandran $\mathrm{C}$ and Melnick SJ: Multidrug resistance in human tumors - molecular diagnosis and clinical significance. Mol Diagn 4: 81-94, 1999.
13. Kondo T, Setoguchi T and Taga T: Persistence of a small subpopulation of cancer stem-like cells in the C6 glioma cell line. Proc Natl Acad Sci USA 101: 781-786, 2004.

14. Harris MA, Yang H and Low BE: Cancer stem cells are enriched in the side population cells in a mouse model of glioma. Cancer Res 68: 10051-10059, 2008.

15. Yanamoto S, Kawasaki G, Yamada S, et al: Isolation and characterization of cancer stem-like side population cells in human oral cancer cells. Oral Oncol 47: 855-860, 2011.

16. Yanamoto S, Kawasaki G, Yoshitomi I, Iwamoto T, Hirata K and Mizuno A: Clinicopathologic significance of EpCAM expression in squamous cell carcinoma of the tongue and its possibility as a potential target for tongue cancer gene therapy. Oral Oncol 43: 869-877, 2007.

17. Goodell MA, Brose K, Paradis G, Conner AS and Mulligan RC: Isolation and functional properties of murine hematopoietic stem cells that are replicating in vivo. J Exp Med 183: 1797-1806, 1996.

18. Diestra JE, Scheffer GL, Català I, et al: Frequent expression of the multi-drug resistance-associated protein $\mathrm{BCRP} / \mathrm{MXR} / \mathrm{ABCP} / A B C G 2$ in human tumours detected by the BXP-21 monoclonal antibody in paraffin-embedded material. J Pathol 198: 213-219, 2002.

19. Lee J, Kotliarova S, Kotliarov Y, et al: Tumor stem cells derived from glioblastomas cultured in bFGF and EGF more closely mirror the phenotype and genotype of primary tumors than do serum-cultured cell lines. Cancer Cell 9: 391-403, 2006.

20. Calabrese C, Poppleton H, Kocak M, et al: A perivascular niche for brain tumor stem cells. Cancer Cell 11: 69-82, 2007.

21. Bleau AM, Hambardzumyan D, Ozawa T, et al: PTEN/PI3K/Akt pathway regulates the side population phenotype and $A B C G 2$ activity in glioma tumor stem-like cells. Cell Stem Cell 4: 226-235, 2009.

22. Gil J, Stembalska A, Pesz KA and Sasiadek MM: Cancer stem cells: the theory and perspectives in cancer therapy. J App Genet 49: 193-199, 2008.

23. Dean M, Fojo T and Bates S: Tumor stem cells and drug resistance. Nat Rev Cancer 5: 275-284, 2004.

24. Eramo A, Ricci-Vitiani L, Zeuner A, et al: Chemotherapy resistance of glioblastoma stem cells. Cell Death Differ 13: 1238-1241, 2006.

25. Zheng X, Shen G, Yang X and Liu W: Most C6 cells are cancer stem cells: evidence from clonal and population analyses. Cancer Res 67: 3691-3697, 2007.

26. Britton KM, Eyre R, Harvey IJ, et al: Breast cancer, side population cells and $A B C G 2$ expression. Cancer Lett 323: 97-105, 2012.

27. Robey RW, Shukla S, Finley EM, Oldham RK, et al: Inhibition of P-glycoprotein $(A B C B 1)$ - and multidrug resistance-associated protein $1(A B C C 1)$-mediated transport by the orally administered inhibitor, CBT-1((R)). Biochem Pharmacol 75: 1302-1312, 2008.

28. Shih AH and Holland EC: Notch signaling enhances nestin expression in gliomas, Neoplasia 8: 1072-1082, 2006.

29. Solecki DJ, Liu XL, Tomoda T, Fang Y and Hatten ME: Activated Notch2 signaling inhibits differentiation of cerebellar granule neuron precursors by maintaining proliferation. Neuron 31: 557-568, 2001.

30. Du Z, Jia D, Liu S, et al: Oct4 is expressed in human gliomas and promotes colony formation in glioma cells. Glia 57: 724-733, 2009. 